\title{
Rethinking Clinical Trials and Personalized Medicine with Placebogenomics and Placebo Dose
}

\author{
Vural Özdemir ${ }^{1}$ and Laszlo Endrenyi ${ }^{2}$
}

\begin{abstract}
Pharmacogenomics, nutrigenomics, vaccinomics, and the nascent field of plant omics are examples of variability science. They are embedded within an overarching framework of personalized medicine. Across these public health specialties, the significance and biology of the placebo response have been historically neglected. A placebo is any substance such as a sugar pill administered in the guise of medication, but one that does not have pharmacological activity. Placebos do have clinical effects, however, that can be substantive in magnitude and vary markedly from person-to-person depending, for example, on the type of disease, symptoms, or clinical trial design. Research over the past several decades attests to a genuine neurobiological basis for placebo effects. All drugs have placebo components that contribute to their overall treatment effect. Placebos are used in clinical trials as control groups to ascertain the net pharmacological effect of a drug candidate. Not only less well known but also relevant to rational therapeutics and personalized medicine is the nocebo. A nocebo effect occurs when an inert substance is administered in a context that induces negative expectations, worsening patients' symptoms. With the COVID-19 pandemic, there are high public expectations for new vaccines and medicines to end the contagion, while at the same time antiscience, post-truth, and antivaccine movements are worrisomely on the rise. These social movements, changes in public health cultures, and conditioned behavioral responses can trigger both placebo and nocebo effects. Hence, in clinical trials, forecasting and explaining placebo and nocebo variability are more important than ever for robust science and personalized health care. Against this overarching context, this article provides (1) a brief history of placebo and (2) a discussion on biology, mechanisms, and variability of placebo effects, and (3) discusses three emerging new concepts: placebogenomics, nocebogenomics, and augmented placebo, that is, the notion of a "placebo dose." We conclude with a roadmap for placebogenomics, its synergies with the nascent field of social pharmacology, and the ways in which a new taxonomy of drug and placebo variability can be anticipated in the next decade.
\end{abstract}

Keywords: placebo variability, placebo dose, placebogenomics, nocebogenomics, augmented placebo, variability science, psychopharmacology

\section{The Power of Words and Expectations}

T HE ACT OF DRUG ADMINISTRATION is embedded in a cultural and sociological context such as patients' expectations, rituals, symbolism, and classical conditioning that invariably accompany and interact with the therapeutic encounter (Fig. 1). Even the sight of a hospital building and physicians' white coats can have effects on physiological measures, including blood pressure and heart rate. Health interventions within caring environments have nonpharmacological corollaries that elicit placebo responses in the person receiving the treatment.
A placebo is any substance such as a sugar pill, saline solution, or sham surgery administered in the guise of medication, but one that is devoid of chemical or pharmacological activity. This definition of placebo does not, however, mean that the placebo intervention is inert clinically. The emerging field of placebo studies and research over the past several decades attest to the presence of a genuine neurobiological basis for the placebo effects (Kaptchuk and Miller, 2015; Schedlowski et al., 2015). Nor are the placebo effects limited to psychological endpoints: "placebo effects that endow inert agents with potency or modify the treatment effects of active pharmacological substances are not only subjective in nature

\footnotetext{
${ }^{1}$ OMICS: A Journal of Integrative Biology, New Rochelle, New York, USA.

${ }^{2}$ Department of Pharmacology and Toxicology, Faculty of Medicine, University of Toronto, Toronto, Canada.
} 


\section{Placebo and Nocebo Effects}

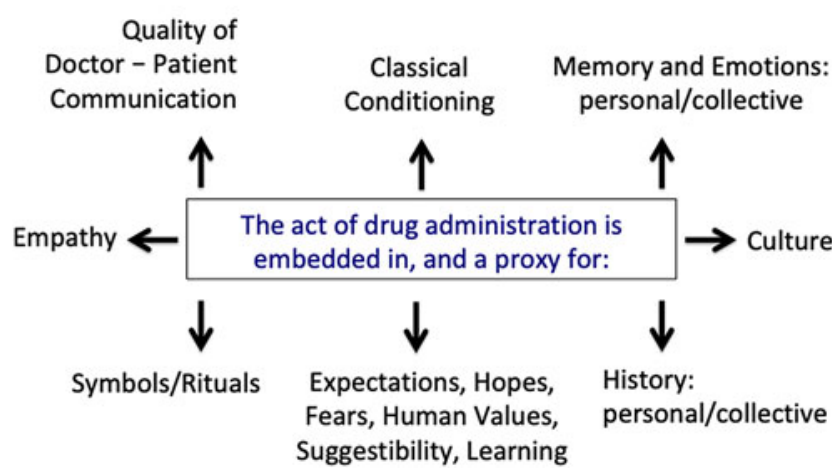

FIG. 1. Nonpharmacological contexts (e.g., expectations, symbolism, associative learning, and classical conditioning) accompany therapeutic encounters in medicine and produce placebo and nocebo effects that modify drug efficacy and side effects, respectively.

[...] they can also be associated with measurable and thus objective changes in end organ functions" (Schedlowski et al., 2015).

The archetypal placebo effect is desirable and leads to an improvement in patients' symptoms. All drugs have placebo components embedded in their overall therapeutic effects, although at varying degrees and depending on the type of disease and clinical endpoint they impact. Placebo effects include the natural history of a disease, but are distinct from and over and on top of changes in patients' clinical conditions due to untreated disease, regression to the mean, and spontaneous remission of symptoms.

Not only less well known but also relevant to rational therapeutics and personalized medicine is the nocebo effect that occurs when an inert substance is administered in a cultural and clinical context that induces negative expectations, worsening patients' symptoms (Hahn, 1997). The latter chemically inert substances are called nocebos.

Placebo versus nocebo distinction is not always clear-cut and can be overlapping. The same pharmacologically inert substance and social context may simultaneously produce desirable placebo and undesirable nocebo effects in the same patient. Placebos and nocebos do not necessarily cure or cause a serious disease such as a broken bone. However, they can significantly add to the overall clinical effects of medicines and health interventions. Moreover, placebo and nocebo effects are dynamic measures, that is, they have magnitudes and time courses, which we name here as placebo-dynamics and nocebo-dynamics, respectively (Fig. 2).

With the COVID-19 pandemic wreaking havoc across the planet, there are high public expectations for new vaccines and medicines to end the contagion. At the same time, global populism, authoritarian governance, and antiscience and antivaccine movements are worrisomely on the rise and threatening progress in science and planetary health (Geiselberger, 2017; LaFraniere and Weiland, 2020; Tyson et al., 2020; Van Norman, 2020). These seismic changes, through hype (positive or negative), associative learning, or conditioned behavioral responses, can have marked impacts on placebo and nocebo effects in clinical trials and medical practice. For new

\section{Drugs and Placebo/Nocebo Dynamics}

\author{
Pharmacological Effect \\ Intensity \& Time Course \\ Placebo/Nocebo Effect \\ Intensity \& Time Course \\ - Placebo-dynamics \\ - Nocebo-dynamics
}

FIG. 2. The placebo and nocebo effects display varied magnitudes and time courses reflected in placebo-dynamics and nocebo-dynamics, respectively. Placebo-dynamics and nocebo-dynamics are veritable phenotypes of interest in the nascent field of placebogenomics and nocebogenomics as efforts to discover biomarkers of placebo variability continue. Note also that pharmacological effects of medicines interact with placebo-dynamics and nocebo-dynamics, ultimately contributing to the apparent dynamic changes in clinical symptoms and signs during pharmacotherapy.

vaccine and drug candidates, this calls for a deeper understanding of placebo and nocebo effects in tandem with their molecular action before they receive regulatory approval and introduced in the clinic and public health practice.

The December special issue of the journal featured plant omics and food engineering as important and hitherto underappreciated dimensions in the field of nutrigenomics. From the standpoint of personalized nutrition, and clinical trials of herbal/traditional medicines, COVID-19 drugs and vaccines, forecasting and explaining placebo and nocebo variability, hold great significance for robust systems science and yet have received much less attention than nutrigenomics, pharmacological drug effects, and pharmacogenomics. Although the placebo effects have been approached in the past as nuisance/noise that confounds the pharmacological action of drugs, forecasting individual variability in placebo effects can help personally tailor, not to mention augment the overall effect of a medicine in clinical practice, and inform clinical trial design, thus paving the way for a more humane, personalized, and empathic patient care and rational drug development.

The present article provides (1) a brief history of placebo and (2) a synopsis on biology, mechanisms, and variability of placebo effects, and (3) discusses three emerging new concepts: placebogenomics, nocebogenomics, and augmented placebo, that is, the notion of a "placebo dose." We conclude with a roadmap for placebogenomics, its synergies with the nascent field of social pharmacology, and the ways in which a new taxonomy of variability science can be anticipated in the next decade.

\section{A Brief History of Placebo}

The first use of placebo in a medical context dates back to the $18^{\text {th }}$ century. Alexander Sutherland, a physician, used the term to critique medial fads of the time such as water cures (Jütte, 2013; Sutherland, 1763). Subsequently, William Cullen, a Scottish physician, mentioned it in connection with administering an ineffective or low-dose drug so as to please the patients (Cullen, 1772; Kerr et al., 2008). This use by Cullen was an active placebo, a substance with pharmacological activity, but not on the disease that is being treated, for example, when a cure is not possible or to satisfy patients' demands for a prescription. John Coakley Lettsom, 
another 18th century British physician, used active placebos in similar contexts (Lettsom, 1772). These early examples and meanings attached to placebo also attest to the importance of the ethics of placebo that continues to challenge medicine, and remains a topic of importance in the $21 \mathrm{st}$ century therapeutics (Raicek et al., 2012).

Placebo was not always used in a medical vein or in a context of positive expectations from health interventions, however. Before the 18th century, in medieval times, the word had a religious connotation. In an early Latin translation of the Hebrew Bible, Psalm 116, verse 9, it was observed as Placebo Domine - "I will please the Lord" (Kerr et al., 2008). The etymology of placebo has been noted as being from the Latin "I shall please" and placere (to please). On the other hand, others (Cai and He, 2019; Lasagna, 1986) have emphasized that the original meaning of the word was "to walk," instead of "to please":

"In the 1300s, the word 'placebo' first appeared in a Latin translation of the Hebrew Bible, with the original meaning of 'to walk' (Lasagna, 1986). Later, because of a mistranslation, placebo was defined as 'to please' (Finniss, 2018). With this positive meaning, the word placebo was recorded as meaning 'to please' in early usage. In the mid-to-late 1700s, the term placebo began to be used as medical jargon (Cai and He, 2019)."

"Historically the term placebo harks back to the 116th Psalm in the Hebrew bible. The ninth verse of this psalm begins with the word "et-ha-lech" (I shall walk), which was strangely translated into the Septuagint Greek as "euarestiso" and then into its Vulgate Latin equivalent of "placebo," the first-person singular future tense of the verb meaning to please [...] A few centuries later, placebo began to take on secular meanings, almost all of them derisive. For example, it was used to denote a servile flatterer.." (Lasagna, 1986).

In the late 19th and early 20th century, the meaning of the word placebo shifted once again, referring to inert substances used in pharmacology experiments to control for "nonspecific" beneficial effects of drugs (Kaptchuk, 1998). Considering that many modern medicines came to prominence and were introduced for clinical use over the last century, the framing of placebo effects as being nonspecific and arguably less significant than the specific molecular effects of drugs is not surprising in a context of the early 20th century.

Onward to the mid-20th century, Stuart Wolf mentioned the placebo effect in 1950, while also presciently hinting both interindividual and intraindividual variability in placebo effects:

"The fact that 'placebo effects' occur depends, of course, on the generalization established repeatedly by numerous workers that the mechanisms of the human body are capable of reacting not only to direct physical and chemical stimulation but also to symbolic stimuli, words and events which have somehow acquired special meaning for the individual. The frequency with which 'placebo effects' would be expected and their magnitude probably vary from person to person and from time to time." (Wolf, 1950).

The sentiment and perception of placebo effects as being nonspecific, unimportant, and mostly a nuisance to be controlled for in clinical trials remained as part of the common parlor and scientific cultures until the last quarter of the 20th century. In the late 1970s, medical literature began to feature, however, studies on the neurobiological basis of placebo effects, the placebo analgesia in particular (Levine et al., 1978), followed by observations dissecting the biology and placebo interface in a wide range of symptoms and clinical contexts, including psychiatry and immunology.

These advances paved the way for placebo studies and their scientific legitimacy over the past decade in particular. We suggest that moving forward to the third decade of the 21st century, we will likely witness, once again, another significant shift in clinical pharmacology and therapeutics, and the culture of medicine and clinical trials, to rethink placebo effects not as nuisance, but as an important tool for critically informed clinical trial design and personalized health care.

\section{Many Placebos}

The interest in the placebo effect and response is increasing as a methodological tool and in medical practice (Frisaldi et al., 2020). While scientists in drug development attempt to minimize placebo effects and placebo-related variability in treatment outcomes, health care workers aim to discover, for each patient, the optimal combination of the clinical context, communication, and the health care culture that enhances the therapeutic effects and decrease the adverse effects of health interventions. In reference to placebo and placebo effects, Macedo et al. (2003) have noted the varied definitions and the lack of a consensus: “A consensus has not yet been found. It seems strange to think that one of the major methodological tools of contemporary clinical pharmacology (placebocontrolled clinical trials) does not have an unequivocal definition.."

A deeper examination of the placebo phenotype is indeed crucial. This is necessary not only for clinical trials and medical practice but also for the emerging studies on genomics basis of placebo response that might illuminate the mechanisms by which individuals differ in their responses to the nonpharmacological context of therapeutics. The nascent fields of social pharmacology and placebo studies offer significant synergy and prospects in this regard so as to understand variability in treatment outcomes from molecules to culture to critical sociology of life sciences (Özdemir, 2020a).

Figure 3 presents the various components of apparent changes in patients' symptoms and clinical signs in the course of a clinical trial or pharmacotherapy in medical practice. The actual placebo effect is elicited by expectations, rituals, associative learning, and conditioning attendant to drug administration after accounting for the natural history of untreated disease. The apparent placebo effect is measured and deduced from the placebo arm of a clinical trial when a chemically inert placebo is used, without a "no-treatment" control group. Hence, the apparent placebo effect is a complex phenotype that reflects both the natural history of untreated disease plus the actual placebo effect. For future genomics and multiomics association studies that aim to uncover the molecular biomarkers of placebo variability, the use of the actual placebo effects as a phenotype of interest would be more relevant and allow for a methodologically astute study design.

An active placebo is a treatment with a chemical activity that mimics the side effects of the drug that is being tested in a clinical trial. Active placebos are sometimes, but not always, used in clinical trials to prevent unblinding of the drug versus the placebo control group (Moncrieff et al., 2004). For example, consider a new hypothetical antidepressant drug candidate in clinical trials that targets depression-related pathways in the brain, while also causing dry mouth as a side 


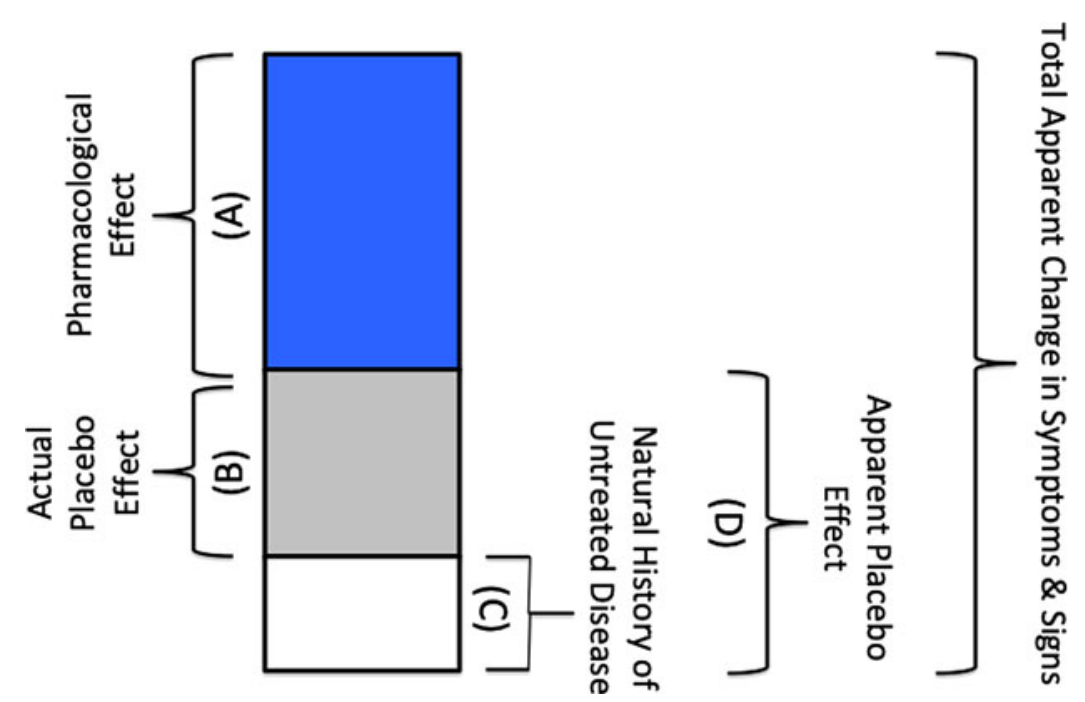

FIG. 3. Dissecting the components of changes in patients' clinical symptoms and signs: (A) the pharmacological effect, produced by a drug's chemical effects on efficacy-related molecular targets, (B) the actual placebo effect, (C) the natural history of untreated disease, regression to the mean and spontaneous remission of symptoms, and (D) apparent placebo effect that includes the actual placebo effect and the natural history of untreated disease, for example, in a clinical trial that employs a chemically inert placebo, without a "no treatment" control group. Note that the relationship between pharmacological and placebo effects is not always additive in nature, and may potentially involve complex nonadditive interactions.

effect. An active placebo that elicits dry mouth as a side effect, but without effects on molecular targets in the brain or those related to depression, would help decrease the probability of unblinding in the course of the clinical trial.

Placebo response refers to placebo effects, neurobiological and psychophysiological responses, elicited by placebo or any active treatment. In the latter context, placebo response is a broader clinical phenotype. All drug and health intervention outcomes have placebo response components as part of their social contexts.

\section{Biology and Mechanisms of Placebo Effect}

Early and seminal studies in biology of placebo effects were conducted in the field of pain research beginning in the 1970s. Levine et al. (1978) provided evidence for the involvement of endogenously released opioids by showing that placeboinduced analgesia can be antagonized by naloxone. A positron emission tomography study showed that both opioid and placebo analgesia are associated with an increased activity in the rostral anterior cingulate cortex, suggesting related neural mechanisms in placebo and drug action (Petrovic et al., 2002). Other biological pathways such as dopamine, cholecystokinin, and endocannabinoids were also implicated in placebo analgesia (Benedetti, 1997; Hall et al., 2015; Scott et al., 2008). More recently, genetic studies began to offer further support for the neurobiology of placebo effects (Hall et al., 2012, 2015; Peciña et al., 2013a, 2013b, 2015).

In particular, and of profound consequence in this moment of the COVID-19 pandemic, are the changes in immune function as a result of placebo effects. Studies in rodents and humans have shown in the past that associative learning and behavioral conditioned responses play a larger role in placebo-mediated changes in peripheral immune function than cognitive factors such as subjects' and patients' expectations (Schedlowski et al., 2015). For example, in a double-blind placebo-controlled study, Goebel et al. (2002) demonstrated the brain-to-immune system interaction. Accordingly, healthy subjects were conditioned over 3 consecutive days while the immunosuppressive drug cyclosporine was administered as an unconditioned stimulus, paired with a uniquely flavored drink (conditioned stimulus). In the subsequent week, reexposure to the drink paired with placebo capsules induced immune function suppression as evidenced by changes in cytokine mRNA expression and lymphocyte proliferation (Goebel et al., 2002).

Similar observations on a brain-to-immune system interaction and learning mechanisms contributing to placebo effects were made in relationship to the allergic symptoms and molecular markers of allergy (e.g., changes in mast cell tryptase) induced in patients in the absence of allergens (Gauci et al., 1994; Turnbull, 1962).

In psychiatry, sleep disorders, and a host of medical conditions, placebo effects and placebo variability play a significant role, not to mention in clinical trials that are charged to develop new therapeutics in these indications. Although placebo effects tend to be more pronounced when measured by subjective or patient-reported symptoms, particularly in psychiatric diseases and clinical psychopharmacology, they do exist to a clinically appreciable extent when assessed with biological indicators of disease as well (Su et al., 2004).

Patients' expectancy of the therapeutic benefits of a medicine as well as conditioning and associative learning are two noteworthy overarching mechanisms mediating the placebo phenotype. Future research is timely and much needed in relationship to the biology of placebo/nocebo effects. In particular, placebo and immune function linkages remain intriguing hypotheses to study in relationship to the current efforts for vaccine and drug development for the COVID-19 pandemic in the face of extensive polarization in planetary society due to rise of global populism and authoritarian governance systems. These changes in governance and society 
may potentially impact the extent and types of associative learning and behavioral conditioning that contribute to peripheral immune function as well as the frequency and intensity of placebo/nocebo effects in COVID-19 clinical trials.

Placebo phenotypes, their kinetics (time course), accrual, and reversal of placebo effects over time and space are subjects worthy of consideration as the new concept of placebogenomics, discussed later in this article, begins to gain traction in medical literature.

The interested reader can find further discussion on biology and mechanisms of placebo effects elsewhere (Benedetti et al., 2005; Cai and He, 2019; Hall et al., 2015, 2018; Schedlowski et al., 2015).

\section{Magnitude and Variability of Placebo Effects}

Placebo effects can attain large magnitudes and vary markedly from person-to-person. This is observed with objective outcome measures as well. A meta-analysis in sleep disorders (primary insomnia) found that $63.56 \%$ of the drug responses are achieved in the placebo groups upon assessment with physiological, polysomnographic parameters (Winkler and Rief, 2015). In active Crohn's disease, metaanalysis of the placebo-controlled randomized clinical trials using as outcome measure the Crohn's Disease Activity Index that incorporates objective and subjective evaluations, placebo response was $19 \%$ (95\% confidence interval, $13 \%-$ 28\%; range, 0\%-46\%) (Su et al., 2004).

The extent to which placebo responses vary and explain treatment outcomes after controlling for the natural history diseases is an important subject that is in need of further research. To do so, a "no treatment" arm in clinical trials is required, but this is often ethically unacceptable, especially in severe disease, which makes the assessment of the "pure" actual placebo effects over and above the natural history of diseases difficult to ascertain.

To quantify and separate placebo effects, spontaneous remission in symptoms, and effect of active treatment, Krogsbøll et al. (2009) analyzed data from 37 trials in 8 clinical conditions (2900 patients). The active health interventions ranged from psychological ( $n=17$ trials) and physical $(n=15$ trials) to pharmacological ( $n=5$ trials). These authors rightly caution against the common and false practice of attributing "the effect that is observed in a placebo arm of a randomized trial as the effect of placebo, as it includes the spontaneous improvement that would also have occurred without administration of a placebo" (Krogsbøll et al., 2009). On the other hand, the latter analysis also shows that placebo effects remain significant and variable after controlling for the natural history of disease: "both the spontaneous improvement and the effect of placebo contribute importantly to the observed treatment effect in actively treated patients, and that the relative importance of these factors differs according to clinical condition and intervention." (Krogsbøll et al., 2009).

Some of the highest placebo responses are noted in clinical trials in the mental health field (Weimar et al., 2015a). Moreover, the true magnitude of a drug effect can be overestimated, and conversely, the placebo effects underestimated when there is a publication bias toward reporting of the positive clinical trial results (Turner et al., 2008). Notwithstanding the caveat that the entire collection of placebo-controlled trials with strong placebo effects and negative results are not always available in the published literature, placebo response rates still remain high in psychiatry (Schedlowski et al., 2015). A meta-analysis found an overall $37 \%$ placebo response rate in antidepressant trials, whereas placebo improvement in depression and anxiety was higher than that in obsessive-compulsive disorder ( $\mathrm{Li}$ et al., 2019), suggesting that the type of diagnosis impacts the placebo effects.

Head-to-head clinical trials with active controls are necessary and called for especially when active treatments with demonstrated efficacy and safety are available. From a placebo studies standpoint, it is noteworthy that the clinical trial designs with multiple active treatment study groups translate to stronger patient expectations and higher probability of receiving the active drug rather than placebo. In effect, this can increase the response rates observed in placebo groups in clinical trials. Consistent with this, Li et al. (2019) reported that "across all indications, placebo response rate was greater in studies with a higher number of active arms." This speaks to the ways in which clinical trial designs contribute to both the magnitude and variability in the observed placebo response rates.

Among the patient-related factors that influence placebo variability, age and sex have often been debated in the literature. On the other hand, there appears to be no consistent evidence that age and sex markedly impact placebo effects, while the type and severity of clinical diagnosis (mild versus severe disease) contribute to placebo variability and size of placebo effect. Weimer et al. (2015b) conducted an evaluation of systematic reviews and meta-analyses for predictors of placebo response across medicine. They found that lower symptom and disease severity at baseline as a patient-related factor, an unbalanced randomization that assigns a greater number of patients to drugs than to placebo, more frequent study visits, and more recent trials were associated with higher placebo response rates.

An interesting observation is the reported increase in placebo response over time in U.S. clinical trials of neuropathic pain (Tutte et al., 2015), which was associated with an increase in study size and length. Although a mechanistic explanation of this observation requires further research, it speaks to the growing importance of placebo studies as a legitimate field of inquiry well beyond the dated and false perception of placebo effects as merely a nuisance to be overcome in clinical trials. Another observation made in the latter study was "different kinetics for the treatment versus placebo responses, with the former evolving more quickly than the latter and plateauing" (Tutte et al., 2015). As we discuss later, the new scholarly fields of placebogenomics and social pharmacology are well poised to make important strides to unravel the biological mechanisms and sociological cues that affect the kinetics of placebo response and what we have named earlier in this article as placebo-dynamics.

Placebo effects certainly have broad significance for research, clinical practice, social pharmacology, and a holistic approach to pharmacotherapy that integrates biology with critical studies in sociology and humanities.

\section{Nocebo Effects}

Nocebo and nocebo effects, in contrast to patients' improvements by placebo effects, have received relatively less attention, but remain as emerging topics of broad scientific interest in pharmacology, medicine, psychology, and social 
sciences. Nocebo effects are noxious effects of a pharmacologically inert substance administered in a negative clinical and social context that create negative expectations in the subject and patients. Hall et al. (2015) defined nocebo effects as "the opposite of placebo effects. They are negative or adverse effects in response to an inert or placebo treatment."

Several variants of nocebo effects exist that clinically manifest as new adverse symptoms, worsening of existing side effects associated with drug or placebo, or as noted by Schedlowski et al. (2015), "the reduced efficacy of clinical interventions due to negative expectations or prior experiences."

Nocebo effects are common in clinical trials. In Parkinson's disease, for example, in a meta-analysis of 3544 placebo-treated patients in randomized controlled trials, at least one adverse drug reaction event (ADR) was reported in 64.7\% (95\% CI: 53.6-74.4) of patients following placebo administration, while $8.8 \%$ (95\% CI: 6.8-11.5) discontinued placebo treatment due to intolerance (Stathis et al., 2013).

Nocebo mechanisms can also contribute to ADRs during switch from brand to generic drugs. In a study purportedly testing the effectiveness of fast-acting $\beta$-blocker medications, an apparent change from brand to generic drug formulation was associated with increased side effects (Faasse et al., 2013).

Media coverage can have positive or negative effects leading to nocebo events as well. Perhaps the most striking and relevant for planetary health at this moment (Van Norman, 2020) for clinical trials of COVID-19 drugs and vaccines are the ways in which an epidemic of adverse events might potentially emerge through nocebo effects. A past example before the COVID-19 era can be instructive in this regard. In New Zealand, an observational study suggested that sensationalized television news coverage of a thyroxin product formulation change might have led to a surge in the overall rate of ADR reporting during the attendant health scare (Faasse et al., 2012).

Historically, there has been a relative asymmetry of research investments in placebo versus nocebo studies. Interindividual and intraindividual variability in nocebo mechanisms, kinetics of nocebo effects, their molecular determinants, and sociological context await further research attention.

\section{What Is Next for Placebo and Nocebo Studies?}

Placebo studies in the mainstream medical research and clinical practice are relatively new, and nocebo studies are even newer. On the other hand, our synopsis on the history and biology of placebo attests to the long-standing efforts to move placebo from a fringe academic curiosity to becoming legitimate bedrock of 21 st century medicine, integrative biology, and health care. For this to happen, future scholars ought to work on both sides of the "two cultures" divide, from life sciences as well as the nascent field of social pharmacology (Özdemir, 2014, 2020a), which examine the social and cultural dynamics, their backstage, and the ways in which the therapeutic encounter and its critical sociology collectively inform treatment outcomes.

Having gained legitimacy with new findings on its neurobiological and neuroimaging substrates, placebo studies have recently expanded toward biomarkers of placebo variability, and genetics/genomics research in particular. Understanding why individuals display marked differences in placebo responses has value for clinical trial design and medical therapeutics. For example, placebo response bio- markers may help explain when drug and placebo effects do not significantly separate due to more frequent representation, by chance, of high placebo responders in small samplesized or nonrandomized clinical trials. In clinical practice, a biomarker panel that predicts both high placebo and high pharmacological effects would allow for personalized use of a drug in patients who can simultaneously benefit from enhanced placebo and pharmacological responses.

The weight of the evidence available to date indicates the role of anticipation as well as associative learning and classical conditioning in placebo responses. Variability in reward-, expectancy-, or "suggestibility"-related neurotransmitter pathways provides a rationale for candidate gene and multiomics studies of placebo variability. Dopamine, opioid, cannabinoid, and serotonin pathways are prominent candidates for genetic studies of placebo phenotypes, and indeed were researched in clinical association studies as reviewed elsewhere (Hall et al., 2015). To this end, placebome refers to "the hypothesized group of genome-related or genome-derived molecules (i.e., genes, proteins, or miRNAs) that affect an individual's response to placebo treatment" (Hall et al., 2015).

The candidate gene approach to study placebo variability has been recently scaled up with network/pathway analyses. Wang et al. (2017) identified that the placebome is rich in neurotransmitter signaling pathways and brain-specific proteins. Interestingly, they report the "network proximity of the placebome module to disease modules in the interactome significantly correlates with the strength of the placebo effect in the corresponding diseases. The proximity of the placebome module to molecular pathways affected by certain drug classes indicates the existence of placebo-drug interactions" (Wang et al., 2017).

Considering that the biological correlates of placebo response such as dopamine, opioid, and serotonin pathways are also well-established molecular drug targets, and in light of the network analyses by Wang et al. (2017), the possibility of placebo-gene-drug interactions raises intriguing prospects and challenges for future clinical trials, pharmacogenomics, and personalized medicine.

For example, pharmacogenomics association studies of drug responders/nonresponders may want to consider analyses of not only drug-treated but also placebo-treated control subjects. This would allow ascertainment of whether the identified pharmacogenomics association findings reflect a genuine biomarker for pharmacological or placebo variability, or both. Moreover, the apparent placebo effect (Fig. 3) as a phenotype of interest is not ideal for it includes the natural history of disease and therefore does not reflect the true extent of placebo variability. A no-treatment control group would be necessary, and yet is not always feasible due to ethical unacceptability unless the study is perhaps conducted in patients with mild disease without the risk of progression.

Finally, the prospect of a placebo-gene-drug interaction means that we need to rethink clinical trial designs as the placebo effects may not always be simply additive to drug effects. Placebo effects embedded in the rituals, expectancies, and symbolism of drug administration is not identical to those observed in the placebo (only) treatment arm of a clinical trial; the latter will be devoid of the placebo-gene-drug interactions. Transferability of placebo biomarkers discovered in clinical trials to placebo variability in medical practice is another challenging issue. These questions are worthwhile to ponder upon as the findings on the placebome continue to accumulate 
in the literature, and are poised to transition for applications in drug development and personalized health care.

\section{Placebogenomics and Nocebogenomics}

Genome-wide and multiomics studies are timely to complement the recent candidate gene and network analyses of placebo response variability. On the other hand, despite patient-related factors, including the clinical indication, symptom severity at baseline, and emerging genetic studies of placebo phenotypes, and research design-related factors such as unbalanced randomization in clinical trials affecting the placebo outcomes (Weimar et al., 2015a), the predictors of the placebo response are multifactorial, vary from one therapeutic field and clinical endpoint to another, and are still to be discovered.

Moving forward in placebo studies, scholarship from variability science can help formulate a strategy roadmap. Variability science has a storied past and examines the mechanisms by which drug, nutrition, vaccine, and health intervention outcomes vary from person to person and between populations. Pharmacogenomics, nutrigenomics, vaccinomics, and the nascent field of plant omics featured in the December issue of OMICS are examples of variability science that are embedded within the overarching framework of personalized medicine (Endrenyi et al., 1976; Kalow, 2001; Loukas et al., 2011; Mishra et al., 2013; Özdemir, 2014). Across these public health specialties, the significance and biology of placebo variability are increasingly noted.

We define here placebogenomics and nocebogenomics, as the multiomics study of interindividual and intraindividual variability in placebo and nocebo phenotypes, respectively.

Intraindividual variability in placebo/nocebo responses has not received adequate attention to date. The within-person variability of placebo and nocebo outcomes is important as the field transitions beyond clinical trials to personalized medicine applications in the future.

Pharmacogenomics is one of the oldest and most established fields of variability science. Placebo variability and potential overlaps among pharmacogenomics, placebogenomics, and nocebogenomics biomarkers indicate the need for commensurate and real-time advances in these fields of inquiry (Fig. 4).

Placebogenomics/nocebogenomics have not been firmly included in a context of pharmacogenomics research in the past. These new fields of scholarship deserve to be additional foundational pillars of research on the role of heredity in drug efficacy and safety. Past research in pharmacogenomics, and particularly in psychiatric pharmacogenomics, ought to be revisited in light of the emerging findings on placebo response biomarkers and because placebo responses tend to be robust in psychiatric pharmacotherapy as discussed earlier.

Pharmacogenomics, placebogenomics, and nocebogenomics employ association study design as a key methodology in human studies. This involves establishing correlations between genomics and multiomics variation versus phenotypic variability, often using sophisticated statistical and bioinformatics analyses. While omics technologies and statistical analyses are fairly well advanced and available, phenotype ascertainment or the field of phenomics is an important bottleneck, as well as an opportunity for progress in placebogenomics and pharmacogenomics (Özdemir, 2020b).

\section{Multi-Omic Dissection of Pharmacological and Placebo Variability}

Pharmacogenomics

Pharmacoproteomics

Pharmacometabolomics

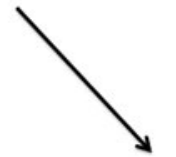

\section{Personalized Medicine}

FIG. 4. As both pharmacogenomics and placebogenomics attain a broader multiomics focus in the near future, they are likely to move on to create new fields of inquiry such as placeboproteomics and placebometabolomics, with an eye to personalized medicine. This would help integrate, triangulate, and validate knowledge streams across pharmacogenomics, placebo studies, social pharmacology, and multiple levels of the biological hierarchy in cell biology.

Most clinical trials employ placebo as a control group to evaluate drug efficacy and safety as a primary goal rather than discovery of placebo response biomarkers. Consequently, the "no-treatment control" groups are not usually included in clinical trial designs. This results in measurement of the apparent placebo effects instead of the actual placebo effects in most clinical trials available to date (Fig. 3). Studies in healthy volunteers can remedy this knowledge gap to some extent, but prospective studies in patient populations that specifically measure the actual placebo phenotypes, in ways that are ethically acceptable and designed with the primary aim of placebo response biomarker discovery, are needed in the future.

There are many placebo phenotypes that vary with the disease and the clinical endpoint in question, social context of pharmacotherapy, and the side effects of medicines. The next section describes a new placebo phenotype, augmented placebo, which might offer new ways forward in placebo variability research.

\section{Placebo Dose-Response and Augmented Placebo}

We propose a new concept and term, augmented placebo, to illustrate and broaden the value of placebos that cause side effects, mimicking the adverse (but not therapeutic) effects of the drug of interest, thus delivering to the patient an "additional dose of expectancy" of therapeutic effect and a sense of "being on the drug arm of the clinical trial," and by extension, a higher magnitude of placebo effect than a regular, chemically inert placebo (Figs. 5 and 6).

There are several rationales in favor of augmented placebo instead of active placebo, as concept and practice, in placebogenomics and clinical trial design. First, active placebo implies that there are inactive placebos. Although a placebo is pharmacologically inert, it does have clinical effects, with neurobiological and neuroimaging substrates. Second, active placebo has been, in general, used in reference to a compound that does not necessarily impact the therapeutic effects of a drug, but mimics its side effects to prevent the loss of blinding in a placebo-controlled clinical trial. By contrast, an 


\section{Concept of Augmented Placebo}

\section{Drug action on a molecular target unrelated to efficacy endpoints and causing adverse drug reaction (ADR)}

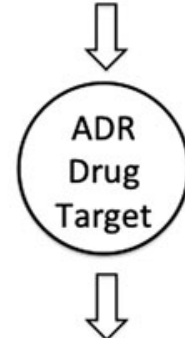

A perception of

"I am on the drug"

by virtue of $A D R$<smiles>C1=CCCC1</smiles>

\section{Augmented Placebo Effect}

FIG. 5. Augmented placebo effect is produced by ADRs/side effects that create an additional perception of being on the drug arm of a blinded clinical trial, over and on top of the symbolism, rituals, etc. noted in Figure 1 for the pharmacologically inert placebo effect. ADR, adverse drug reaction.

augmented placebo, by virtue of the side effects it produces, allows for a more granular and graded understanding, for example, the possibilities of low and high placebo doses, than the traditional chemically inert placebo. Third, augmented placebo is a broader term that accommodates the social cues and cultural triggers that can also lead to placebo doseresponse relationships, mediated, for example, by the color of the drug capsule, drug price, and branding or marketing intensity (see, for examples, Meissner and Linde, 2018).

Looking at from the perspective of drugs, most drugs have side effects and in this sense, drug treatment outcomes in clinical practice are a product of pharmacological activity on efficacy targets, the augmented placebo effect elicited by drug side effects, plus the regular placebo effect (Fig. 6), and the complex ways in which these components interact. Augmented placebo, therefore, speaks to the notion of a placebo dose and the necessity to consider it in clinical trial designs and personalizing health care.

It is obvious that the concept of placebo dose is, and will be, different than the dose range of a precisely measurable chemical entity that defines a drug dose. However, a combination of factors, including medication side effects,

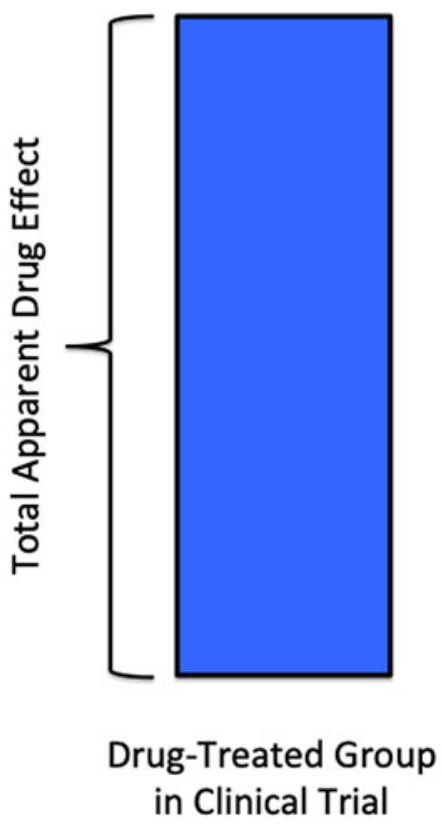

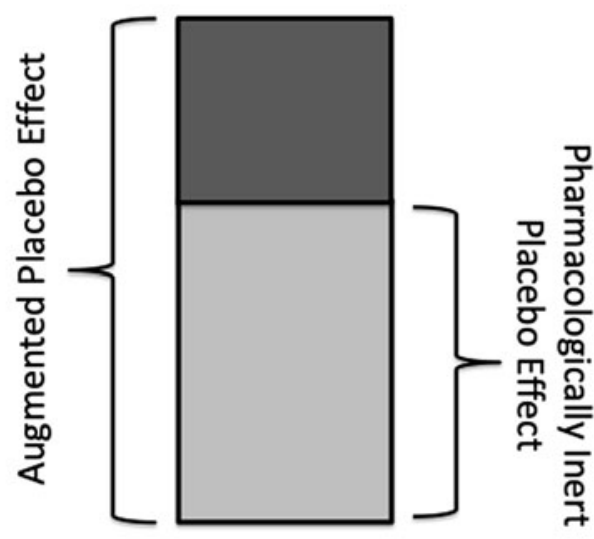

\section{Placebo-Treated Group} in Clinical Trial

FIG. 6. Augmented placebo effect includes and extends beyond the pharmacologically inert placebo effect, reducing the outcome difference between drug and placebo arms of a clinical trial. 
physical attributes like the color and size of the drug capsule, invasiveness and route of administration (e.g., oral vs. subcutaneous placebo), social and cultural milieu of the physician's office, and the health care facility might, in aggregate, create an ordinal rank of placebo intensities or placebo doseresponse with differential intensities. Several lines of evidence already support the idea of augmentation of placebo effects through medication side effects.

Moncrieff et al. (2004) conducted a pooled analysis of the controlled clinical trials of antidepressants that have used placebos that produce and mimic the antidepressant side effects. The difference between drug and placebo in their analysis was small compared to studies that do not select for pharmacologically active placebos. They suggest that "the effects of antidepressants may generally be overestimated and their placebo effects may be underestimated," that is, if placebos are chemically inert and unmatched for drug side effects in clinical trials.

Indeed, medication effects, therapeutic or adverse, may prevent the complete blinding of the drug treatment group in clinical trials. In a randomized, double-blind comparison of alprazolam, imipramine, and placebo, Margraf et al. (1991) report that "the great majority of panic disorder patients $(N=59)$ and their physicians were able to rate accurately whether active drug or placebo had been given."

Further evidence for augmented placebo effects and placebo dose comes from an experimental study in healthy volunteers (Rief and Glombiewski, 2012). A nasal placebo spray that induces a prickling nasal sensation (sesame oil with $0.014 \%$ capsaicin) was used to mimic the side effects of a painkiller, and compared with an inert nasal spray (sesame oil). When the subjects were advised they have a 50\% chance to receive the drug, the pain threshold was substantially higher in the group that received the nasal placebo spray with the prickling sensation compared to the inert placebo group.

Thus far, we have considered the pharmacotherapies without a pharmacogenomics companion diagnostic test to individually tailor the medication dose and/or prescription. In personalized medicine, however, the parallel information (and expectancy) stream from the pharmacogenomics test results can result in additional placebo and nocebo effects that could shape the placebo dose-response relationships (Fig. 7). Haga et al. (2009) have aptly noted the potential of placebo/nocebo effects in pharmacogenomics. In theory, epigenomics factors can contribute to intraindividual variability in placebo doseresponse relationships together with ever-changing social and cultural dynamics enacting on patients' anticipation of medication therapeutic effects and/or ADRs.

Taken together, the placebo dose and augmented placebo effects have relevance to (1) evaluation of the net pharmacological effects of drugs in clinical trials and (2) how we measure placebo phenotypes in studies of the genomics and multiomics basis of placebo variability. At present, three placebo-related phenotypes are conceivably possible in placebogenomics research: apparent placebo effect, actual placebo effect, and the augmented placebo effect (Figs. 3 and 6).

\section{A New Taxonomy of Placebo Response and Variability?}

There are many placebo phenotypes with complex and heterogeneous molecular, sociological, cultural, and psy-

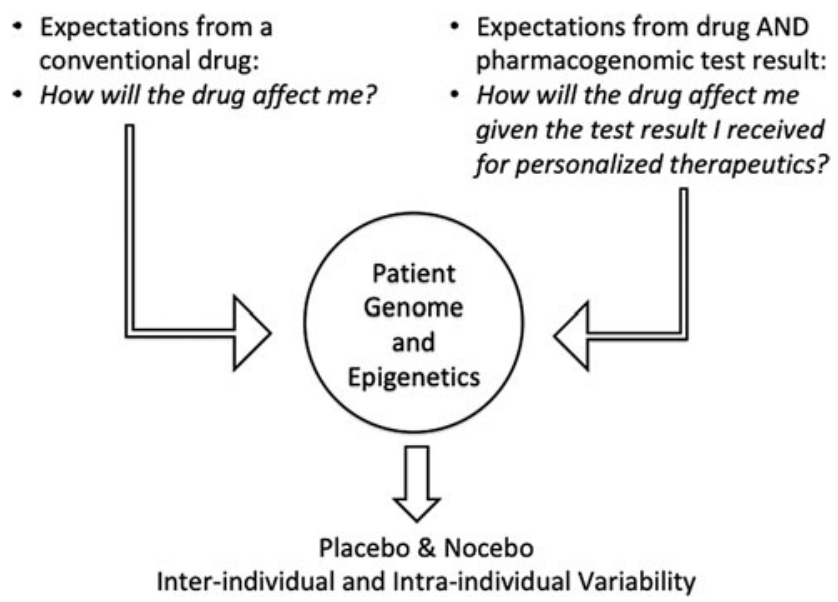

FIG. 7. Additional expectancy and associative learning/conditioning can be triggered in patients by pharmacogenomics test results in the field of personalized medicine, in connection with and over and on top of the regular expectations from the drug (Right side of the figure). This can potentially trigger complex placebo/nocebo effects interacting with the pharmacological and placebo/nocebo effects from the drug. This contrasts with the treatment by a conventional drug that is not personalized and used without a pharmacogenomics test (Left side of the figure).

chological mechanisms. As new evidence accumulates along these pillars, new taxonomies to classify placebo phenotypes will be possible. For example, the two general mechanisms often mentioned in the development of placebo phenotypes, expectation and conditioning, likely have their comprehensive molecular correlates whose identification will contribute to future taxonomies driven by the mechanistic architecture of placebo responses.

Past approaches to placebo tended to classify placebo responders versus nonresponders. On the other hand, the placebo dose-response relationship as well as the kinetics (time course) of placebo responses could serve as phenotypes of interest in placebogenomics, rather than the binary of placebo responders/nonresponders. In the field of pharmacogenomics, the shifts in drug dose versus response curves and the kinetics of drug response are often utilized as highly granular phenotypes. Why not use placebo dose or early and late placebo responders or the time course of placebo response as nuanced placebo phenotypes in placebogenomics research?

Genomics, epigenomics, and multiomics variability can impact drug and placebo outcomes at multiple levels (Fig. 8). Emerging data in placebo studies suggest that we may need to revisit our assumption of additive effects between placebo and pharmacological effects and the ways in which they interact with disease and genomics variability.

A major consideration that has been hitherto overlooked in genomics studies of placebo outcomes is that the heritability of each placebo phenotype will vary depending on the drug, clinical endpoint, and disease, to name but a few caveats. As an alternative to the twin method, our group has developed in the 1990s a high-throughput statistical approach, a repeated drug administration (RDA) method, to measure the genetic components in pharmacological traits (Kalow et al., 1998; Ozdemir et al., 2000). The RDA method may presumably inform placebogenomics study designs in the future. 


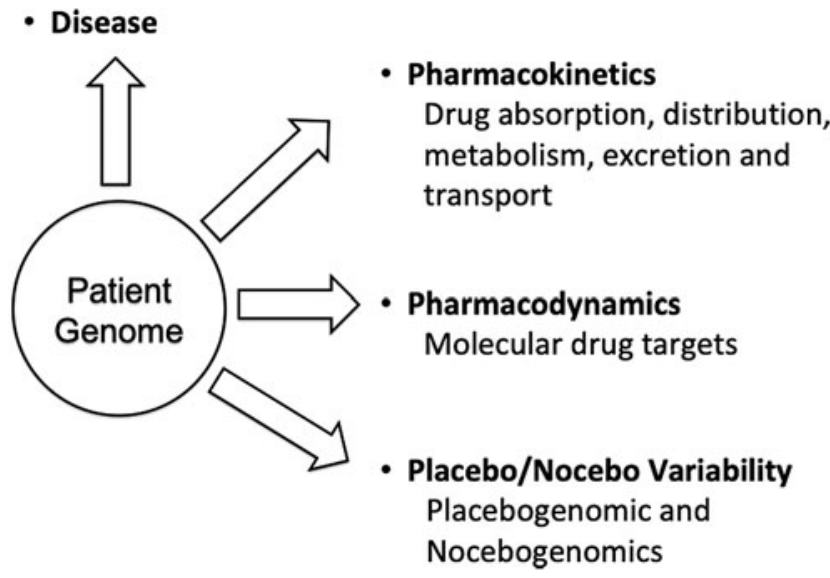

FIG. 8. Genomics, epigenomics, and multiomics variations, among patients and populations, can influence (1) disease susceptibility and prognosis, (2) drug pharmacokinetics, (3) drug efficacy targets, and (4) placebo/nocebo effects.

Moreover, heritability of pharmacological traits can display time dependency as shown in the case of the CYP3A4 drugmetabolizing enzyme (Ozdemir et al., 2000). Prioritizing placebogenomics studies in highly heritable placebo phenotypes that are measured in optimal clinical and temporal contexts could then enhance the chance of identifying robust biomarkers of placebo variability. Figure 9 presents a suggestion for an initial placebogenomics strategy and roadmap along these lines.

\section{Concluding Thoughts}

Drugs and health care produce measurable clinical effects not only by pharmacological action but also through placebo and nocebo effects mediated by social and cultural circumstances. Placebo studies have begun to illuminate the neurobiological mechanisms underlying placebo and nocebo responses. The appreciable magnitude and the interindividual variability of placebo effects attest to their importance in personalized health care, clinical trials, and research. The nascent fields of placebogenomics, nocebogenomics, and social pharmacology offer the promise of unraveling the biomarkers and social determinants of placebo and nocebo variability. They also raise sociological and ethical challenges in the way clinical trials are designed, risk information is communicated, and placebo responses are harnessed in health care.

Everyday, millions of individuals receive genetic and other diagnostic test results for common and rare human diseases. Interestingly, in a recent study, Turnwald et al. (2019) noted that "merely receiving genetic risk information changed individuals' cardiorespiratory physiology, perceived exertion and running endurance during exercise, and changed satiety physiology and perceived fullness after food consumption in a self-fulfilling manner. Effects of perceived genetic risk on outcomes were sometimes greater than the effects associated with actual genetic risk. If simply conveying genetic risk information can alter actual risk, clinicians and ethicists should wrestle with appropriate thresholds for when revealing genetic risk is warranted." Corollary of these observations is that the placebo and nocebo effects attendant to pharmacogenomics test results warrant further research and consideration in personalized medicine.

What and who are excluded matter greatly (Barad, 2011). Sometimes, what are excluded and pushed to the sidelines are certain fields of scholarship (Özdemir, 2020a). Placebo and nocebo studies, and their offspring subspecialties placebogenomics and nocebogenomics, examine placebo and nocebo variability and warrant legitimate investments in mainstream medicine and modern therapeutics. Moreover, the currently evolving COVID-19 pandemic is likely a "dry run" for future ecological crises such as climate change and other zoonotic outbreaks (Özdemir, 2020c; Springer, 2020). Ecological and planetary health crises invariably result in heightened public expectations, fears, and uncertainties that can fuel placebo and nocebo effects in drug and vaccine development. Seen in this light, placebogenomics and nocebogenomics are valuable and timely additions to 21 st century medicine. Their contributions

\section{Phenomics as Bottleneck \& Development Strategy}

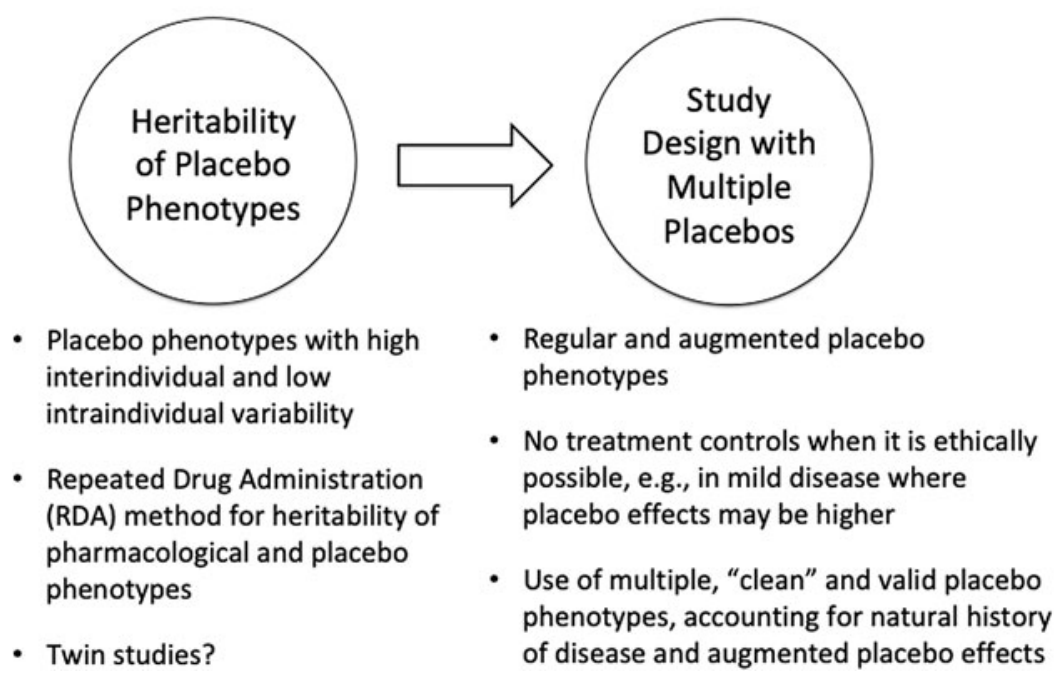

FIG. 9. Phenomics is both a bottleneck and strategy/solution focus in placebogenomics R\&D. Placebo phenotypes need to be considered, both in concept and practice, before multiomics studies, for example, candidate gene or genome-/proteome-/ metabolome-wide inquiries, are designed. $\& \& D$, research and development. 
are not only limited to research but also might help realize a humane health care that recognizes individual variability in the way we respond to health interventions. Therefore, we conclude the article with philosopher Plato, born circa 428423 BC, and teachings in Ancient Greece (Papakostas and Daras, 2001) from Charmides, a dialog of Plato:

Socrates: "Good doctors ... (it) is not possible for them to attempt to treat the head by itself, apart from the body ... or the body without the soul ... and the treatment of the soul by means of certain charms, and these charms are words of the right sort ..." (Plato, 1927)

\section{Disclaimer}

The views expressed are the personal opinions of the authors only.

\section{Author Disclosure Statement}

The authors declare they have no conflicting financial interests.

\section{Funding Information}

No funding was received in support of this analysis.

\section{References}

Barad K. (2011). Erasers and erasures: Pinch's unfortunate “uncertainty principle." Soc Stud Sci 41, 443-454.

Benedetti F. (1997). Cholecystokinin type-A and type-B receptors and their modulation of opioid analgesia. News Physiol Sci 12, 263-268.

Benedetti F, Mayberg HS, Wager TD, Stohler CS, and Zubieta JK. (2005). Neurobiological mechanisms of the placebo effect. J Neurosci 25, 10390-10402.

Cai L, and He L. (2019). Placebo effects and the molecular biological components involved. Gen Psychiatr 32, e100089.

Cullen W. (1772). Cullen Clinical Lectures 1772-1773. Edinburgh: RCPE Manuscript, Cullen, 218-219.

Endrenyi L, Inaba T, and Kalow W. (1976). Genetic study of amobarbital elimination based on its kinetics in twins. Clin Pharmacol Ther 20, 701-714.

Faasse K, Gamble G, Cundy T, and Petrie KJ. (2012). Impact of television coverage on the number and type of symptoms reported during a health scare: A retrospective pre-post observational study. BMJ Open 2, e001607

Faasse K, Cundy T, Gamble G, and Petrie KJ. (2013). The effect of an apparent change to a branded or generic medication on drug effectiveness and side effects. Psychosom Med 75, 90-96.

Finniss DG. (2018). Placebo effects: Historical and modern evaluation. Int Rev Neurobiol 139, 1-27.

Frisaldi E, Shaibani A, and Benedetti F. (2020). Understanding the mechanisms of placebo and nocebo effects. Swiss Med Wkly 150 , w20340.

Gauci M, Husband AJ, Saxarra H, and King MG. (1994). Pavlovian conditioning of nasal tryptase release in human subjects with allergic rhinitis. Physiol Behav 55, 823-825.

Geiselberger H. (2017). The Great Regression. Cambridge, United Kingdom: Polity Press.

Goebel MU, Trebst AE, Steiner J, et al. (2002). Behavioral conditioning of immunosuppression is possible in humans. FASEB J 16, 1869-1873.
Haga SB, Warner LR, and O'Daniel J. (2009). The potential of a placebo/nocebo effect in pharmacogenetics. Public Health Genomics 12, 158-162.

Hahn RA. (1997). The nocebo phenomenon: Scope and foundations. In: The Placebo Effect: An Interdisciplinary Exploration. Harrington A, ed. Cambridge, MA: Harvard University Press, $56-76$.

Hall KT, Lembo AJ, Kirsch I, et al. (2012). Catechol-Omethyltransferase val158-met polymorphism predicts placebo effect in irritable bowel syndrome. PLoS One 7, e48135.

Hall KT, Loscalzo J, and Kaptchuk TJ. (2015). Genetics and the placebo effect: The placebome. Trends Mol Med 21, 285-294.

Hall KT, Loscalzo J, and Kaptchuk T. (2018). Pharmacogenomics and the Placebo Response. ACS Chem Neurosci 9, 633-635.

Jütte R. (2013). The early history of the placebo. Complement Ther Med 21, 94-97.

Kalow W, Tang BK, and Endrenyi L. (1998). Hypothesis: Comparisons of inter- and intra-individual variations can substitute for twin studies in drug research. Pharmacogenetics 8, 283-289.

Kalow W. (2001). Pharmacogenetics in perspective. Drug Metab Dispos 29(4 Pt 2), 468-470.

Kaptchuk TJ. (1998). Intentional ignorance: A history of blind assessment and placebo controls in medicine. Bull Hist Med 72, 389-433.

Kaptchuk TJ, and Miller FG. (2015). Placebo Effects in Medicine. N Engl J Med 373, 8-9.

Kerr C, Milne I, and Kaptchuk TJ. (2008). William Cullen and a missing mind-body link in the early history of placebos. J R Soc Med 101, 89-92.

Krogsbøll LT, Hróbjartsson A, and Gøtzsche PC. (2009). Spontaneous improvement in randomized clinical trials: Metaanalysis of three-armed trials comparing no treatment, placebo and active intervention. BMC Med Res Methodol 9, 1.

LaFraniere S, and Weiland N. (2020). White House Blocks New Coronavirus Vaccine Guidelines. The New York Times, October 5. https://www.nytimes.com/2020/10/05/us/politics/coronavirusvaccine-guidelines.html?action=click \&module=Spotlight \&pg type $=$ Homepage. Accessed October 27, 2020.

Lasagna L. (1986). The placebo effect. J Allergy Clin Immunol $78,161-165$.

Lettsom JC. (1772). Reflections on the General Treatment and Cure of Fevers. London: J.D. Cornish.

Levine JD, Gordon NC, and Fields HL. (1978). The mechanisms of placebo analgesia. Lancet 2, 654-657.

Li F, Nasir M, Olten B, and Bloch MH. (2019). Meta-Analysis of Placebo Response in Adult Antidepressant Trials. CNS Drugs 33, 971-980.

Loukas A, Gaze S, Mulvenna JP, et al. (2011). Vaccinomics for the major blood feeding helminths of humans. OMICS 15, 567-577.

Macedo A, Farre M, and Banos J-E. (2003). Placebo effect and placebos: What are we talking about? Some conceptual and historical considerations. Eur J Clin Pharmacol 59, 337-342.

Margraf J, Ehlers A, Roth WT, et al. (1991). How "blind" are double-blind studies? J Consult Clin Psychol 59, 184-187.

Meissner K, and Linde K. (2018). Are Blue Pills Better Than Green? How Treatment Features Modulate Placebo Effects. Int Rev Neurobiol 139, 357-378.

Mishra M, Kanwar P, Singh A, Pandey A, Kapoor S, and Pandey GK. (2013). Plant omics: Genome-wide analysis of ABA repressor1 (ABR1) related genes in rice during abiotic stress and development. OMICS 17, 439-450. 
Moncrieff J, Wessely S, and Hardy R. (2004). Active placebos versus antidepressants for depression. Cochrane Database Syst Rev 1, CD003012.

Ozdemir V, Kalow W, Tang BK, eT al. (2000). Evaluation of the genetic component of variability in CYP3A4 activity: A repeated drug administration method. Pharmacogenetics 10, 373-388.

Özdemir V. (2014). Personalized medicine across disciplines and without borders. Per Med 11, 687-691.

Özdemir V. (2020a). Why are some omics biotechnologies more popular than others? the sociomateriality of glycans offers new clues. OMICS 24, 57-59.

Özdemir V. (2020b). Phenomics 2.0: Real-world real-time patient outcomes measured by the internet of pharmaceutical things. OMICS 24, 119-121.

Özdemir V. (2020c). "One Nature": A new vocabulary and frame for governance innovation in post-COVID-19 planetary health. OMICS 24, 645-648.

Papakostas YG, and Daras MD. (2001). Placebos, placebo effect, and the response to the healing situation: The evolution of a concept. Epilepsia 42, 1614-1625.

Plato XII. (Lamb, WRM transl.) (1927). Charmides, Alcibiades I and II, Hipparchus, The Lovers, Theages, Minos, Epinomis. Cambridge, MA: Harvard University Press.

Peciña M, Azhar H, Love TM, et al. (2013a). Personality trait predictors of placebo analgesia and neurobiological correlates. Neuropsychopharmacology 38, 639-646.

Peciña M, Stohler CS, and Zubieta JK. (2013b). Role of $\mathrm{m}$-opioid system in the formation of memory of placebo responses. Mol Psychiatry 18, 135-137.

Peciña M, Love T, Stohler CS, Goldman D, and Zubieta JK. (2015). Effects of the $\mathrm{Mu}$ opioid receptor polymorphism (OPRM1 A118G) on pain regulation, placebo effects and associated personality trait measures. Neuropsychopharmacology 40, 957-965.

Petrovic P, Kalso E, Petersson KM, and Ingvar M. (2002). Placebo and opioid analgesia - imaging a shared neuronal network. Science 295, 1737-1740.

Raicek JE, Stone BH, and Kaptchuk TJ. (2012). Placebos in 19th century medicine: A quantitative analysis of the BMJ. BMJ 345, e8326.

Rief W, and Glombiewski JA. (2012). The hidden effects of blinded, placebo-controlled randomized trials: An experimental investigation. Pain 153, 2473-2477.

Schedlowski M, Enck P, Rief W, and Bingel U. (2015). Neurobio-behavioral mechanisms of placebo and nocebo responses: implications for clinical trials and clinical practice. Pharmacol Rev 67, 697-730.

Scott D, Stohler C, Egnatuk C, Wang H, Koeppe R, and Zubieta JK. (2008). Placebo and nocebo effects are defined by opposite opioid and dopaminergic responses. Arch Gen Psychiatry $65,220-231$.

Springer S. (2020). Caring geographies: The COVID-19 interregnum and a return to mutual aid. Dialogues Hum Geography $10,112-115$.

Stathis P, Smpiliris M, Konitsiotis S, and Mitsikostas DD. (2013). Nocebo as a potential confounding factor in clinical trials for Parkinson's disease treatment: A meta-analysis. Eur J Neurol 20, 527-533.

Su C, Lichtenstein GR, Krok K, Brensinger CM, and Lewis JD. (2004). A meta-analysis of the placebo rates of remission and response in clinical trials of active Crohn's disease. Gastroenterology 126, 1257-1269.

Sutherland A. (1763). Attempts to Revive Ancient Medical Doctrines: I. Of Waters in General [..]. London: A. Millar.
Turnbull JW. (1962). Asthma conceived as a learned response. J Psychosom Res 6, 59-70.

Turner EH, Matthews AM, Linardatos E, Tell RA, and Rosenthal R. (2008). Selective publication of antidepressant trials and its influence on apparent efficacy. N Engl J Med 358, 252-260.

Turnwald BP, Goyer JP, Boles DZ, Silder A, Delp SL, and Crum AJ. (2019). Learning one's genetic risk changes physiology independent of actual genetic risk. Nat Hum Behav 3, 48-56.

Tuttle AH, Tohyama S, Ramsay T, et al. (2015). Increasing placebo responses over time in U.S. clinical trials of neuropathic pain. Pain 156, 2616-2626.

Tyson A, Johnson C, and Funk C. (2020). U.S. Public Now Divided Over Whether To Get COVID-19 Vaccine. Pew Research Center, September 17. https://www.pewresearch.org/science/ 2020/09/17/u-s-public-now-divided-over-whether-to-get-covid19-vaccine/. Accessed October 27, 2020.

Van Norman GA. (2020). "Warp Speed" Operations in the COVID-19 Pandemic: Moving Too Quickly? ACC Basic Transl Sci 5, 730-734

Wang RS, Hall KT, Giulianini F, Passow D, Kaptchuk TJ, and Loscalzo J. (2017). Network analysis of the genomic basis of the placebo effect. JCI Insight 2, e93911.

Weimer K, Colloca L, and Enck P. (2015a). Placebo effects in psychiatry: Mediators and moderators. Lancet Psychiatry 2, 246-257.

Weimer K, Colloca L, and Enck P. (2015b). Age and sex as moderators of the placebo response - an evaluation of systematic reviews and meta-analyses across medicine. Gerontology 61, 97-108.

Winkler A, and Rief W. (2015). Effect of placebo conditions on polysomnographic parameters in primary insomnia: A metaanalysis. Sleep 38, 925-931.

Wolf S. (1950). Effects of suggestion and conditioning on the action of chemical agents in human subjects; the pharmacology of placebos. J Clin Invest 29, 100-109.

Address correspondence to: Vural Özdemir, $M D, P h D$ Editor-in-Chief OMICS: A Journal of Integrative Biology New Rochelle, NY 10801

USA

E-mail: ojib@liebertpub.com; vural.ozdemir@protonmail.com

and

Laszlo Endrenyi, PhD Professor Emeritus

Department of Pharmacology and Toxicology Faculty of Medicine University of Toronto Toronto M5S $2 A 1$

Canada

E-mail: 1.endrenyi@utoronto.ca

\section{Abbreviations Used}

$\mathrm{ADR}=$ adverse drug reaction

$\mathrm{RDA}=$ repeated drug administration 